\title{
Cytomegalovirus Disease in Renal Transplanted Patients: Prevalence, Determining Factors, and Influence on Graft and Patients Outcomes
}

\author{
Carlo Maria Alfieri ${ }^{1,2, *,+}{ }^{\oplus}$, Paolo Molinari ${ }^{1,+}$, Mariateresa Gandolfo ${ }^{1}$, Mariarosaria Campise ${ }^{1}$, Donata Cresseri ${ }^{1}$, \\ Anna Regalia ${ }^{1}$, Evaldo Favi ${ }^{2,3}$, Min Li $\left.{ }^{4}{ }^{(}\right)$, Masami Ikehata ${ }^{4}\left(\mathbb{D}\right.$, Serena Delbue ${ }^{5}$ and Piergiorgio Messa $^{1,2}$
}

1 Department of Nephrology, Dialysis and Renal Transplantation, Fondazione IRCCS Ca' Granda Ospedale Policlinico Milan, 20122 Milano, Italy; paolo.molinari1@unimi.it (P.M.); mariateresa.gandolfo@policlinico.mi.it (M.G.); maria.campise@policlinico.mi.it (M.C.); donata.cresseri@policlinico.mi.it (D.C.); anna.regalia@policlinico.mi.it (A.R.); piergiorgio.messa@unimi.it (P.M.)

2 Department of Clinical Sciences and Community Health, University of Milan, 20122 Milano, Italy; evaldo.favi@unimi.it

3 Renal Transplantation, Fondazione IRCCS Ca' Granda Ospedale Policlinico Milan, 20122 Milano, Italy

4 Renal Research Laboratory Fondazione IRCCS Ca' Granda Ospedale Policlinico Milan, 20122 Milano, Italy; li_min@libero.it (M.L.); masami.ikehata@policlinico.mi.it (M.I.)

5 Department of Biomedical, Surgical and Dental Sciences, Università degli Studi di Milano, 20122 Milan, Italy; serena.delbue@unimi.it

check for updates

Citation: Alfieri, C.M.; Molinari, P. Gandolfo, M.; Campise, M.; Cresseri, D.; Regalia, A.; Favi, E.; Li, M.; Ikehata, M.; Delbue, S.; et al. Cytomegalovirus Disease in Renal Transplanted Patients: Prevalence, Determining Factors, and Influence on Graft and Patients Outcomes. Pathogens 2021, 10, 473. https:// doi.org/10.3390/pathogens10040473

Academic Editor: Sarah Jackson

Received: 13 March 2021

Accepted: 12 April 2021

Published: 14 April 2021

Publisher's Note: MDPI stays neutral with regard to jurisdictional claims in published maps and institutional affiliations.

Copyright: (c) 2021 by the authors. Licensee MDPI, Basel, Switzerland. This article is an open access article distributed under the terms and conditions of the Creative Commons Attribution (CC BY) license (https:/ / creativecommons.org/licenses/by/ $4.0 /)$.
* Correspondence: carlo.alfieri1@gmail.com; Tel.: +39-02550-34552; Fax: +39-02550-34550

+ These authors have contributed equally to this work.

\begin{abstract}
The prevalence and the factors related to cytomegalovirus (CMV) disease (CMVd) during the 1st year of renal transplantation (RTx) and the relationship between CMVd and early and longterm graft and RTx-patient (RTx-p) survival were evaluated. In 505 RTx-p, followed up for 8(5-11) years, data were recorded after 1-(T1) and 12-(T12) months of RTx. CMVd was defined either by CMV replication without clinical signs of disease (CMVr, 43\%), or CMV replication with signs of disease (CMVs, 57\%). During the 1st year of RTx, 45\% of RTx-p had CMVd (CMVd+). CMVd+ patients were older than CMVd- patients. Female gender and Donor CMV-IgG+ (CMV IgG-D+)/recipient IgG- (CMV IgG-R-) status were more prevalent in CMVd+. At T1, CMVd+ had lower albumin, haemoglobin, and higher uric-acid and reactive $\mathrm{C}$-protein than $\mathrm{CMVd}-$ and, at $\mathrm{T} 1$ and $\mathrm{T} 12$, received more steroids. Albumin-T1 was the unique factor in determining $\mathrm{CMVd}+$, maintaining its significance also after the inclusion of $\operatorname{IgG}-\mathrm{D}+/ \mathrm{IgG}-\mathrm{R}-$ status to the model. CMVs had higher prevalence of CMV IgG-D+/IgG-R- than CMVr. CMVd, CMVr, and CMVs had no impact on graft loss (11\% of RTx-p) and RTx-p death (8\% of RTx-p). CMVd is highly prevalent during the 1st year of RTx. Albumin-T1 influences CMVd insurgence. CMVd did not impact on RTx and RTx-p loss.
\end{abstract}

Keywords: cytomegalovirus; infection; renal transplantation; graft outcome; albumin

\section{Introduction}

The prevalence of chronic kidney disease (CKD) has increased in recent years and renal transplantation (RTx) is considered the best therapy. Despite this, RTx patients (RTx-p) are characterized by a high risk of complications, partly related to their clinical characteristics and to therapies prescribed during the RTx. In this scenario, infectious diseases have a strong impact on graft and patient survival [1-3]

Cytomegalovirus (CMV) disease has a high prevalence in RTx-p, especially during the first year of RTx, ranging between 10\% and 42\% [4,5]. Generally, CMV disease (CMVd) may be classified according to the presence of clinical and biochemical signs in: asymptomatic CMV replication (CMVr) and symptomatic CMV replication (CMVs) [6]. Data present in 
literature have hypothesized a potential impact of CMVd on the negative outcome of RTx-p and RTx [7-9].

In our retrospective observational study, we evaluated the prevalence and the most important factors related to CMVd, the association between CMVd, and the principal long-term clinical outcomes in a cohort of RTx-p followed-up in our Department.

\section{Results}

\subsection{Cohort Characteristics}

The population examined in this study was composed of 505 RTx-p, of which Table 1 summarizes the main clinical characteristics.

Table 1. Main general characteristics of the cohort studied.

\begin{tabular}{|c|c|}
\hline Parameters & \\
\hline $\begin{array}{c}\text { Number of patients } \\
\mathbf{N}\end{array}$ & 505 \\
\hline Age at RTx (years) & $50(41-58)$ \\
\hline $\begin{array}{c}\text { Gender }(M-F) \\
\mathbf{N}\end{array}$ & $292-213$ \\
\hline $\begin{array}{c}\text { Native kidney disease } \\
\text { N (\%) } \\
\text { GNF } \\
\text { GNC } \\
\text { ADPKD } \\
\text { OTHER } \\
\text { CNP } \\
\text { NDD } \\
\text { VASCULITES } \\
\text { UES }\end{array}$ & $\begin{array}{c}106(21) \\
94(18) \\
92(18) \\
89(18) \\
49(10) \\
46(9) \\
26(5) \\
3(1)\end{array}$ \\
\hline $\begin{array}{c}\text { Dialysis type (HD-PD-No) } \\
\mathbf{N} \\
(\%) \\
\end{array}$ & $\begin{array}{c}372-105-28 \\
(74-21-5)\end{array}$ \\
\hline Dialysis vintage (months) & $52(37-76)$ \\
\hline $\begin{array}{c}\text { RTx type } \\
\text { (deceased-living donor) } \\
\mathbf{N} \\
(\mathbf{\%})\end{array}$ & $\begin{array}{l}458-47 \\
(91-9)\end{array}$ \\
\hline $\begin{array}{c}\text { Number of RTx } \\
(1-2-3) \\
\mathbf{N} \\
(\%)\end{array}$ & $\begin{array}{l}412-89-4 \\
(81-18-1)\end{array}$ \\
\hline Cold ischemia time (hours) & $13(11-16)$ \\
\hline $\begin{array}{c}\text { Diabetes at RTx } \\
\text { N } \\
(\%)\end{array}$ & $\begin{array}{l}23 \\
(4)\end{array}$ \\
\hline $\begin{array}{c}\text { Steroid therapy before RTx } \\
\mathbf{N} \\
(\%)\end{array}$ & $\begin{array}{l}189 \\
(38)\end{array}$ \\
\hline
\end{tabular}


Table 1. Cont.

\begin{tabular}{cc}
\hline Parameters & \\
\hline CMV Receiving-Donor Serology & \\
N (\%) & $17(3)$ \\
CMV R-/D- & $64(13)$ \\
CMV R-/D+ & $52(10)$ \\
CMV R+/D- & $372(74)$ \\
CMV R+/D+ & \\
HCV+ before RTx & 31 \\
N & $(6)$ \\
\hline
\end{tabular}

Note: N: Number; RTx: renal transplantation; M: male; F: female; GNF: glomerulonephritis; GNC: chronic glomerulonephritis; ADPKD; autosomal dominant polycystic kidney disease; CNP: chronic pyelonephritis; NDD: non determined disease; UES: uremic emolytic syndrome; HD: hemodialysis; PD: peritoneal dialysis; CMV: cytomegalovirus; R: recipient; D: donor; HCV: hepatitis C virus.

The cohort was composed mainly of males $(\mathrm{n}=292)$ and the median age of the cohort was 50 (41-58) years. Most RTx-p received hemodialysis (HD) before RTx (74\%), while only $21 \%$ were treated with peritoneal dialysis (PD). Ninety-one percent of RTx-p received a kidney from a deceased donor. Among the 47 RTx-p who received a RTx from a living donor, 12 were transplanted from a related donor.

As shown in Table 2, in most cases, immunosuppressive induction therapy was composed of basiliximab and steroids ( $87 \%$ ). The maintenance immunosuppressive therapy included calcineurin inhibitors, mainly Tacrolimus $(88 \%)$, mycophenolate/mycophenolic acid $(95 \%)$, and steroids $(92 \%)$. At T1, only $3 \%$ of patients were treated with $\mathrm{m}$-Tor inhibitors. This distribution showed no substantial differences at T12.

Table 2. Immunosuppression therapy of the cohort studied.

\begin{tabular}{ccc}
\hline Drugs & T1 & T12 \\
\hline \multicolumn{3}{c}{ Immunosuppressive induction therapy } \\
\hline Basiliximab N (\%) & $442(87)$ \\
ATG N (\%) & $58(11)$ & NA \\
No Basiliximab-No ATG N (\%) & $5(2)$ & \\
\hline \multicolumn{3}{c}{ Maintenance immunosuppressive therapy } \\
\hline MMF-MPA-Tac-Steroids-CyA-m-Tor-I & $443-479-463-60-15$ & $440-407-458-56-34$ \\
N (\%) & $(95-88-92-12-3)$ & $(80-87-91-12-7)$ \\
\hline Cumulative dose of steroids (mg) & $880(840-1050)$ & $2722(2598-3223)$ \\
\hline
\end{tabular}

Note: N: number; ATG: antithymoglobuline; CyA: Ciclosporine; MMF-MPA: mycophenolate-mycophenolic acid; Tac: tacrolimus; m-Tor-I: m-Tor inhibitors.

In Table 3, the main anthropometric and biochemical characteristics of the cohort studied at $\mathrm{T} 1$ and $\mathrm{T} 12$ are reported.

During the first year of RTx, a significant increase of body weight $(p<0.001)$ was found. No significant modification in blood pressure control was demonstrated.

Concerning renal function, serum creatinine $(\mathrm{sCr})$ and daily uriniary protein excretion (Prot-U) were similar at T1 and T12. However, a significant reduction of estimated glomerular filtration rate (eGFR) $(p<0.0001)$ was found. In addition, a significant increase of uric acid, hemoglobin $(\mathrm{Hb})$, serum albumin, and glycosylate hemoglobin $(\mathrm{HbA} 1 \mathrm{C})($ all $p<0.0001$ ) was observed. 
Table 3. Anthropometric and biochemical characteristics of the cohort studied.

\begin{tabular}{cccc}
\hline Parameters & T1 & T12 & $p$ \\
\hline Body Weight (Kg) & $65(56-73)$ & $68(57-75)$ & $<0.001$ \\
\hline SBP (mmHg) & $130(120-140)$ & $130(120-140)$ & 0.7 \\
\hline DBP (mmHg) & $80(75-90)$ & $80(75-85)$ & 0.59 \\
\hline s-Creatinine (mg/dL) & $1.38(1.09-1.7)$ & $1.33(1.1-1.61)$ & 0.21 \\
\hline Prot-U (g/24h) & $0.203(0.143-0.300)$ & $0.175(0.113-0.251)$ & 0.43 \\
\hline eGFR (mL/min) & $53(40-67)$ & $51(41-63)$ & $<\mathbf{0 . 0 0 0 1}$ \\
\hline Uric acid (mg/dL) & $5.8(4.8-6.9)$ & $6.4(5.5-7.5)$ & $<\mathbf{0 . 0 0 0 1}$ \\
\hline Hb (g/dL) & $11(10.05-12)$ & $12.7(11.8-13.8)$ & $<\mathbf{0 . 0 0 0 1}$ \\
\hline s-Albumin (g/dL) & $4.2(4.4-4.5)$ & $4.5(4.2-4.7)$ & $<\mathbf{0 . 0 0 0 1}$ \\
\hline Blood glucose (mg/dL) & $82(72-93)$ & $82(74-94)$ & 0.54 \\
\hline HbA1C (\%) & $5.5(5.1-5.9)$ & $5.7(5.4-6.1)$ & $<\mathbf{0 . 0 0 0 1}$ \\
\hline CRP (mg/dL) & $0.25(0.1-0.64)$ & $0.13(0.1-0.390)$ & $\mathbf{0 . 0 0 1}$ \\
\hline
\end{tabular}

Note: SBP: systolic blood pressure; DBP: diastolic blood pressure; s-: serum; Prot-U: Proteinuria; eGFR: estimated glomerular filtration rate; $\mathrm{Hb}$ : hemoglobin; $\mathrm{HbA1C}$ : glycosylated hemoglobin; CRP: C-reactive protein.

CMV Evaluation

At the time of RTx, the most prevalent serological combination was CMV R+/D+ (74\%), while CMV R-/D+ was present in 13\% of RTx-p.

During the first year of RTx, CMVd was found in 225 RTx-p (45\% of the cohort studied). Tables 4 and 5 illustrate the main differences between patients who experienced $\mathrm{CMVd}$ $(\mathrm{CMVd}+)$ compared to those who did not $(\mathrm{CMVd}-)$ during the first year of $\mathrm{RTx}$.

Table 4. General characteristics of the cohort studied according to CMVd status.

\begin{tabular}{cccc}
\hline Parameters & CMVd+ (N = 225) & CMVd- (N = 280) & $p$ \\
\hline Age (years) & $53(45-59)$ & $47(39-57)$ & $<\mathbf{0 . 0 0 0 1}$ \\
\hline Dialysis vintage (months) & $48(26-75)$ & $51(36-71)$ & 0.103 \\
\hline Cold ischemia (hours) & $13(11-16)$ & $14(11-16)$ & 0.266 \\
\hline Gender M-F & $115-110$ & $177-103$ & $\mathbf{0 . 0 0 6 2}$ \\
\hline Native kidney disease, N & & & \\
GNF & 46 & 59 & \\
ADPN & 42 & 49 & \\
OTHER & 43 & 53 & \\
GNC & 40 & 24 & \\
CNP & 25 & 17 & $\mathbf{0 . 0 1}$ \\
NDD & 19 & 2 & $<0.0001$ \\
VASCULITIS & 9 & $201-56-23$ & $\mathbf{0 . 0 2 3}$ \\
UES & 1 & $233-47$ & 0.889 \\
\hline Dialysis type (HD-PD-No) & $171-49-5$ & $218-58-4$ & 0.412 \\
\hline RTx type (deceased-living) & $225-0$ & $248-32$ & \\
\hline Number of RTx (1-2-3) & $193-32-0$ & $244-36-0$ & \\
\hline BAS-ATG Induction Therapy & $194-26$ & & \\
\hline Calcineurin Inhibitors T1 & $199-25-1$ & & \\
(Tac-Cya-No) & & & \\
\hline
\end{tabular}


Table 4. Cont.

\begin{tabular}{|c|c|c|c|}
\hline Parameters & $\mathrm{CMVd}+(\mathrm{N}=225)$ & CMVd- $(\mathrm{N}=280)$ & $p$ \\
\hline T1 MMF (Yes-No) & $218-7$ & $261-19$ & 0.070 \\
\hline T1 m-Tor-I (Yes-No) & $3-222$ & $14-266$ & 0.015 \\
\hline $\begin{array}{l}\text { Calcineurin Inhibitors T12 } \\
\text { (Tac-Cya-No) }\end{array}$ & $189-29-7$ & $233-34-13$ & 0.746 \\
\hline T12 MMF (Yes-No) & $209-16$ & $250-30$ & 0.132 \\
\hline T12 m-Tor-I (Yes-No) & $13-212$ & $25-255$ & 0.284 \\
\hline $\begin{array}{c}\text { Diabetes at RTx }(\text { Yes-No) } \\
\text { N } \\
(\%)\end{array}$ & $\begin{array}{l}18-207 \\
(8-92)\end{array}$ & $\begin{array}{c}6-274 \\
(2-98)\end{array}$ & 0.0046 \\
\hline $\begin{array}{l}\text { Steroid therapy before RTx } \\
\text { (Yes-No) } \\
\mathbf{N} \\
(\%)\end{array}$ & $\begin{array}{l}88-137 \\
(39-61)\end{array}$ & $\begin{array}{l}118-162 \\
(42-58)\end{array}$ & 0.343 \\
\hline $\begin{array}{c}\text { HCV+ before RTx (Yes-No) } \\
\text { N } \\
(\%)\end{array}$ & $\begin{array}{l}14-211 \\
(6-94)\end{array}$ & $\begin{array}{l}22-258 \\
(8-92)\end{array}$ & 0.576 \\
\hline $\begin{array}{l}\text { CMV Recipient/Donor Serolog } \\
\text { N } \\
\text { CMV R-/D- } \\
\text { CMV R-/D+ } \\
\text { CMV R+/D- } \\
\text { CMV R+/D+ }\end{array}$ & $\begin{array}{c}4 \\
38 \\
18 \\
165\end{array}$ & $\begin{array}{c}13 \\
26 \\
34 \\
207\end{array}$ & 0.01 \\
\hline
\end{tabular}

Note: N: number; M: male; F: female; GNF: glomerulonephritis; ADPKD: autosomal dominant polycystic kidney disease; GNC: chronic glomerulonephritis; CNP: chronic pyelonephritis; NDD: non determined disease; UES: uremic emolytic syndrome; HD: hemodialysis; PD: peritoneal dialysis; RTx: renal transplantation; BAS: Basiliximab; ATG: antithymoglobuline; Tac: Tacrolimus; CyA: Ciclosporine; MMF-MPA: mycophenolate-mycophenolic acid; m-Tor-I: m-Tor inhibitors; HCV: hepatitis C virus; CMV: cytomegalovirus; R: recipient; D: donor.

CMVd+ were older $(p<0.0001)$ and had a higher prevalence of diabetes before RTx $(p=0.004)$. In addition, in this group of RTx-p, the serological status CMV R-/D+ was significantly more represented $(p=0.01)$. Of note, CMVd- was significantly more prevalent in pre-emptive RTx-p $(p=0.01)$. No difference between the two groups was found in relation to dialysis vintage. RTx-p transplanted from a living donor were entirely represented in the CMVd- group.

Immunosuppressive therapy showed no associations with CMVd+ status, except for the cumulative dose of steroids, which was higher in the CMVd+ group, both at T1 $(p=0.006)$ and T12 $(p<0.0001)$.

At T1, the eGFR value was significantly lower in CMVd+ patients $(p=0.006)$. No difference was found in Prot-U. In addition, $\mathrm{CMVd}+$ patients had significantly lower values of $\mathrm{Hb}(p=0.02)$ and serum albumin $(p<0.0001)$, and higher values of uric acid $(p=0.01)$ and CRP $(p=0.008)$ at T1.

During the first year of RTx, the sCr showed no significant variation in either CMVd+ or CMVd- groups (CMVd+ T1: 1.39 (1.1-1.77)-T12: 1.39 (1.1-1.71) mg/dL $p=0.39$; CMVd-T1: $1.37(1.07-1.67)-T 12: 1.30(1.08-1.58) \mathrm{mg} / \mathrm{dL} p=0.36)$. In the same period, in both groups, the eGFR significantly changed (CMVd+ T1: 49 (38-64) - T12: 49 (37-60) $\mathrm{mL} / \mathrm{min} p<0.0001$; CMVd- T1: 55 (44-69-T12: 53 (43-65) $\mathrm{mL} / \mathrm{min} p=0.006$ ). 
Table 5. Anthropometric and biochemical characteristics of the cohort studied according to CMVd status.

\begin{tabular}{cccc}
\hline Parameters T1 & CMVd+ (N 225) & CMVd- (N 280) & $p$ \\
\hline Body weight (Kg) & $65(55-73)$ & $64.5(56.7-73.05)$ & 0.846 \\
\hline SBP (mmHg) & $130(120-145)$ & $130(120-140)$ & 0.819 \\
\hline DBP (mmHg) & $80(70-90)$ & $80(75-90)$ & 0.471 \\
\hline s-Creatinine (mg/dL) & $1.39(1.1-1.77)$ & $1.37(1.07-1.67)$ & 0.225 \\
\hline Prot-U (g/24h) & $0.198(0.140-0.304)$ & $0.210(0.151-0.299)$ & 0.624 \\
\hline eGFR (mL/min) & $49(38-64)$ & $55(44-69)$ & $\mathbf{0 . 0 0 6}$ \\
\hline Uric acid (mg/dL) & $6.1(5.0-7.2)$ & $5.7(4.7-6.7)$ & $\mathbf{0 . 0 1 5}$ \\
\hline Hb (g/dL) & $10.80(9.9-11.8)$ & $11.1(10.3-12.1)$ & $\mathbf{0 . 0 2 6}$ \\
\hline s-Albumin (g/dL) & $4.2(3.9-4.3)$ & $4.3(4.03-4.50)$ & $<\mathbf{0 . 0 0 0 1}$ \\
\hline $\begin{array}{c}\text { Glycemia (mg/dL) } \\
\text { HbA1C (\%) }\end{array}$ & $84(72-94)$ & $81(71.25-93)$ & 0.123 \\
\hline CRP (mg/dL) & $5.5(5.1-5.97)$ & $5.45(5-5.8)$ & 0.125 \\
\hline $\begin{array}{c}\text { the first year of } \\
\text { transplantation (mg) }\end{array}$ & $(840.00-1125.00)$ & $870(835-995)$ & $\mathbf{0 . 0 0 8}$ \\
\hline Cumulative steroids during & & $\mathbf{0 . 0 0 6}$
\end{tabular}

Note: SBP: systolic blood pressure; DBP: diastolic blood pressure; s-: serum-; Prot-U: proteinuria; eGFR: estimated glomerular filtration rate; $\mathrm{Hb}$ : hemoglobin; HbA1C: glycosylated hemoglobin; CRP: C-reactive protein.

In the multivariate analyses shown in Tables 6 and 7, in which age at RTx, T1 serum albumin, $\mathrm{Hb}$ at $\mathrm{T} 1$, and cumulative dose of steroids at $\mathrm{T} 1$ were considered as dependent variables, only the serum albumin at $\mathrm{T} 1$ was independently and inversely correlated with the development of CMVd ( $p=0.009-\mathrm{OR} 0.50-\mathrm{IC}$ 0.29-0.84). To confirm the strength of this relationship, it should be noted that the inclusion in the same model of CMV R-/D+ serologic status, which was also strongly and independently related with $\mathrm{CMVd}$, did not significantly influence the association between serum albumin and CMVd (albumin T1: $p=0.008$-OR 0.49—IC 0.28-0.82; serologic asset CMV R-D+: $p=0.01-\mathrm{OR}$ 2.16-IC 1.18-3.95).

Table 6. Multivariate analysis for the event CMVd+.

\begin{tabular}{cccccc}
\hline Parameter & Coefficient & Standard Error & Odds Ratio & CI & $p$ \\
\hline $\begin{array}{c}\text { Age at } \\
\text { transplantation }\end{array}$ & 0.01 & 0.008 & 1.01 & $0.99-1.03$ & 0.11 \\
\hline s-Albumin T1 & -0.69 & 0.26 & 0.50 & $0.29-0.84$ & $\mathbf{0 . 0 0 9}$ \\
\hline Hb T1 & 0.01 & 0.07 & 1.01 & $0.87-1.17$ & 0.85 \\
\hline Steroids T1 & 0.0004 & 0.0002 & 1.0 & $1.0-1.0$ & 0.06 \\
\hline
\end{tabular}

Note: CI: confidence interval; s-: serum; Hb: hemoglobin.

During the first year of RTx, among CMVd+ subjects, CMVr was reported in 97 patients ( $43 \%$ ), while 128 patients $(57 \%)$ have been considered to be suffering from CMVs.

Among $\mathrm{CMVs}$, on medical indication, specific antiviral treatment was prescribed in $91 \%$ of cases, while, in $63 \%$ of cases, a significant CMV related reduction of immunosuppressive therapy was made. Of note, in $54 \%$ of cases, the two therapeutic approaches coexisted. $\mathrm{CMVr}$ and $\mathrm{CMVs}$ groups were different only in the cumulative dose of steroids during the first year of RTx, significantly higher in CMVs (CMVr: 2722 (2595-3512) mg vs. CMVs 2902 (2674-3782) mg, $p=0.02$ ). 
Table 7. Multivariate analysis for the event CMVd+ including CMV serology at the time of transplantation.

\begin{tabular}{cccccc}
\hline Parameter & Coefficient & Standard Error & Odds Ratio & CI & $p$ \\
\hline $\begin{array}{c}\text { Age at } \\
\text { transplantation }\end{array}$ & 0.014 & 0.009 & 1.014 & $0.99-1.03$ & 0.122 \\
\hline s-Albumin T1 & -0.718 & 0.271 & 0.487 & $0.28-0.82$ & $\mathbf{0 . 0 0 8}$ \\
\hline Hb T1 & -0.0005 & 0.075 & 0.999 & $0.86-1.15$ & 0.994 \\
\hline Steroids T1 & 0.0003 & 0.0002 & 1.0004 & $0.999-1.00$ & 0.084 \\
\hline $\begin{array}{c}\text { CMV serology } \\
\text { D+/R- }\end{array}$ & 0.77 & 0.30 & 2.16 & $1.18-3.95$ & $\mathbf{0 . 0 1}$ \\
\hline
\end{tabular}

Note: CI: confidence interval; s-: serum; Hb: hemoglobin; CMV: cytomegalovirus; D: donor; R: recipient.

\subsection{Principal Outcomes}

During the follow-up time, 58 patients (11\%) re-started dialysis $(\mathrm{D}+)$, and 43 patients $(8 \%)$ died. At $\mathrm{T} 1$ and $\mathrm{T} 12$, the $\mathrm{sCr}$ and Prot- $\mathrm{U}$ values were significantly higher in $\mathrm{D}+(\mathrm{sCr}$ T1: $p<0.0001-T 12: p=0.003$; Prot-U T1: $p=0.004-T 12: p<0.0001)$. Consensually, at T1 and T12, eGFR was significantly lower in D+ (eGFR T1: $p=0.02-\mathrm{T} 12: p=0.01)$. Finally, at $\mathrm{T} 1$ and T12, D+ had higher levels of uric acid (both $p<0.0001)$ and lower of $\mathrm{Hb}(\mathrm{Hb} \mathrm{T} 1$ : $p=0.03-\mathrm{T} 12: p=0.04)$. The serum albumin, on the other hand, showed lower values only at $\mathrm{T} 12$ in $\mathrm{D}+(p=0.02)$.

At the end of follow up, sCr $>50 \%$ was found in 18\% of RTx-p and eGFRr $>50 \%$ in $15 \%$ of RTx-p; In the overall cohort, the median eGFR variation during the FU was $-0.08(-1.44 /+1.11) \mathrm{mL} / \mathrm{min} /$ year.

\subsection{Principal Long Term Outcome Related to CMVd}

As shown in Table 8, no difference in graft failure and combined outcomes was found in relation to CMVd status.

Table 8. Evaluation of graft failure and combined outcomes was found in relation to CMVd status.

\begin{tabular}{|c|c|c|c|}
\hline Parameters & $\mathrm{CMVd}+(\mathrm{N}=225)$ & CMVd- $(\mathrm{N}=280)$ & $p$ \\
\hline \multicolumn{4}{|l|}{$\mathrm{D}(\%)$} \\
\hline No & 204 & 243 & 0.20 \\
\hline Yes & 21 & 37 & \\
\hline \multicolumn{4}{|l|}{$\mathrm{sCr}>50 \%+\mathrm{D}+$} \\
\hline No & 183 & 220 & 0.4672 \\
\hline Yes & 42 & 60 & \\
\hline \multicolumn{4}{|l|}{ eGFRr $>50 \%$ + D+ } \\
\hline No & 197 & 228 & 0.0751 \\
\hline Yes & 28 & 52 & \\
\hline $\begin{array}{l}\text { eGFR: Variation/year of FU } \\
(\mathrm{mL} / \mathrm{min} / \text { year })\end{array}$ & $-0.7(-1.2 /+1.1)$ & $-0.9(-1.6 /+1.1)$ & 0.66 \\
\hline
\end{tabular}

Note: N: number; eGFRr: estimated glomerular filtration rate reduction; sCr: serum creatinine; D: dialysis; D+: dialysis restart.

This result was also confirmed by the analysis of eGFR variation during the FU in the two groups (CMVd+: $-0.7(-1.2 /+1.1) \mathrm{mL} / \mathrm{min} /$ year vs. CMVd-: $-0.9(-1.6 /+1.1)$ $p=0.66$ ).

With the aim to evaluate the possible influence of a primary infection and of a reactivation in the principal outcome, a sub-analysis was performed. Globally, no statistical differences were found between those patients who experienced a first CMV infection and those who had CMV reactivation: D+ 7\% vs. 9\% $p=0.77 ;$ eGFRr $>50 \%+$ D+ $11 \%$ vs. $12 \% p=0.96$ and death $D+7 \%$ vs. $9 \% p=0.77$. Additionally, considering the two subgroups alone, no impact of CMVd type was found. Briefly, in patients 
CMV R-, a first infection compared to no CMV infection did not influenced the main outcome considered: D+ $7 \%$ vs. $9 \% p=056$; eGFRr $>50 \%+D+11 \%$ vs. $13 \% p=0.96$ and death $\mathrm{D}+7 \%$ vs. $10 \% p=0.57$. In patients CMV R+, a CMV reactivation compared to no CMV reactivation did not influence the main outcome considered: D+ $9 \%$ vs. $13 \% p=026$; eGFRr $>50 \%+D+12 \%$ vs. $19 \% p=0.06$ and death $D+7 \%$ vs. $9 \%$ $p=0.38$.

Survival analyses showed no influence of CMVd, CMVr, and CMVs on long-term RTx survival and on RTx-p survival (Figures 1 and 2).

CMVd-, CMVd+ and graft loss

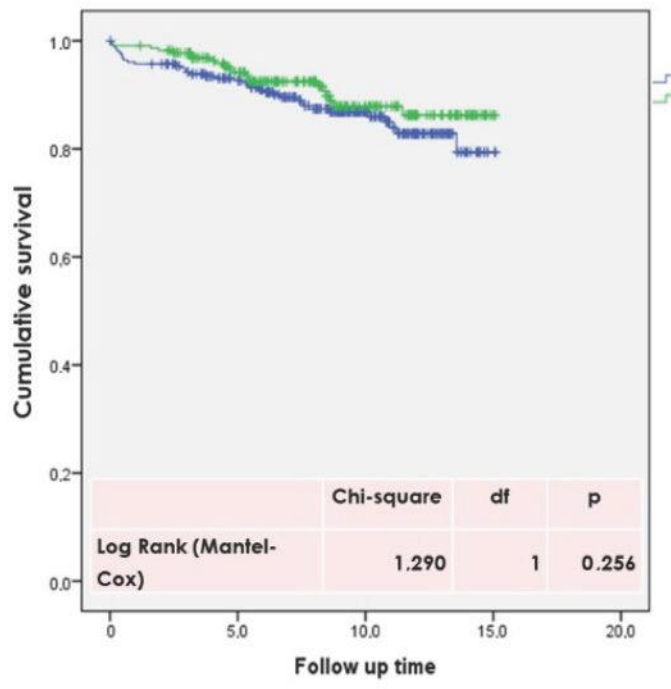

CMVd-, CMVr, CMVs and graft loss
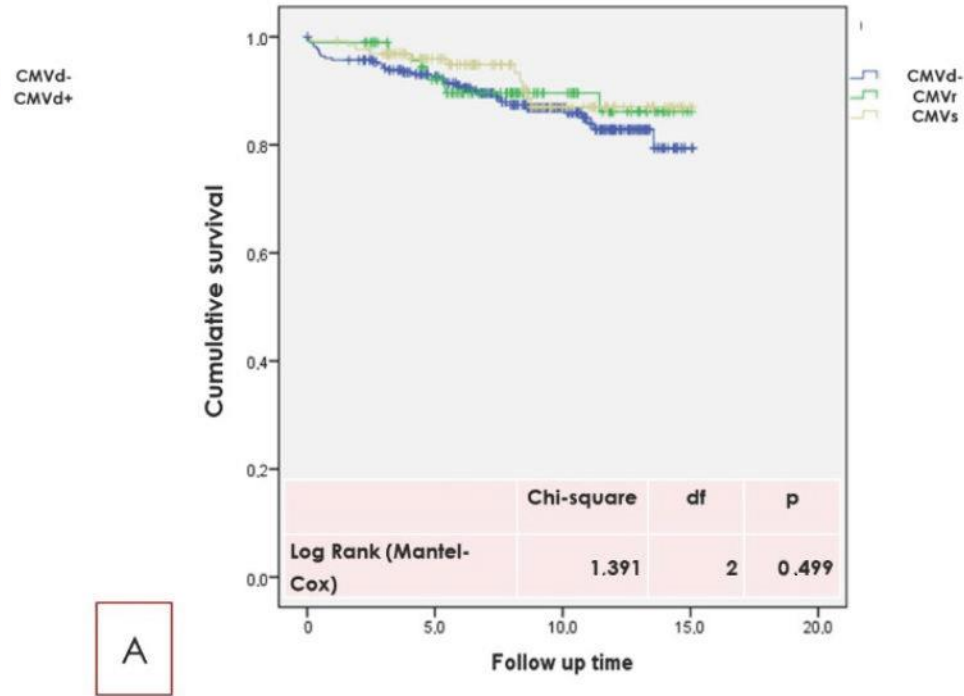

Figure 1. (A): CMVd and graft loss. (B): CMVd-, CMVr, and CMVs and graft loss. As reported in the text, no significant differences for CMVd, CMVe, and CMVs were found according to graft loss experience. Kaplan Meier survival analysis. The log rank test (Mantel-Cox) was used.

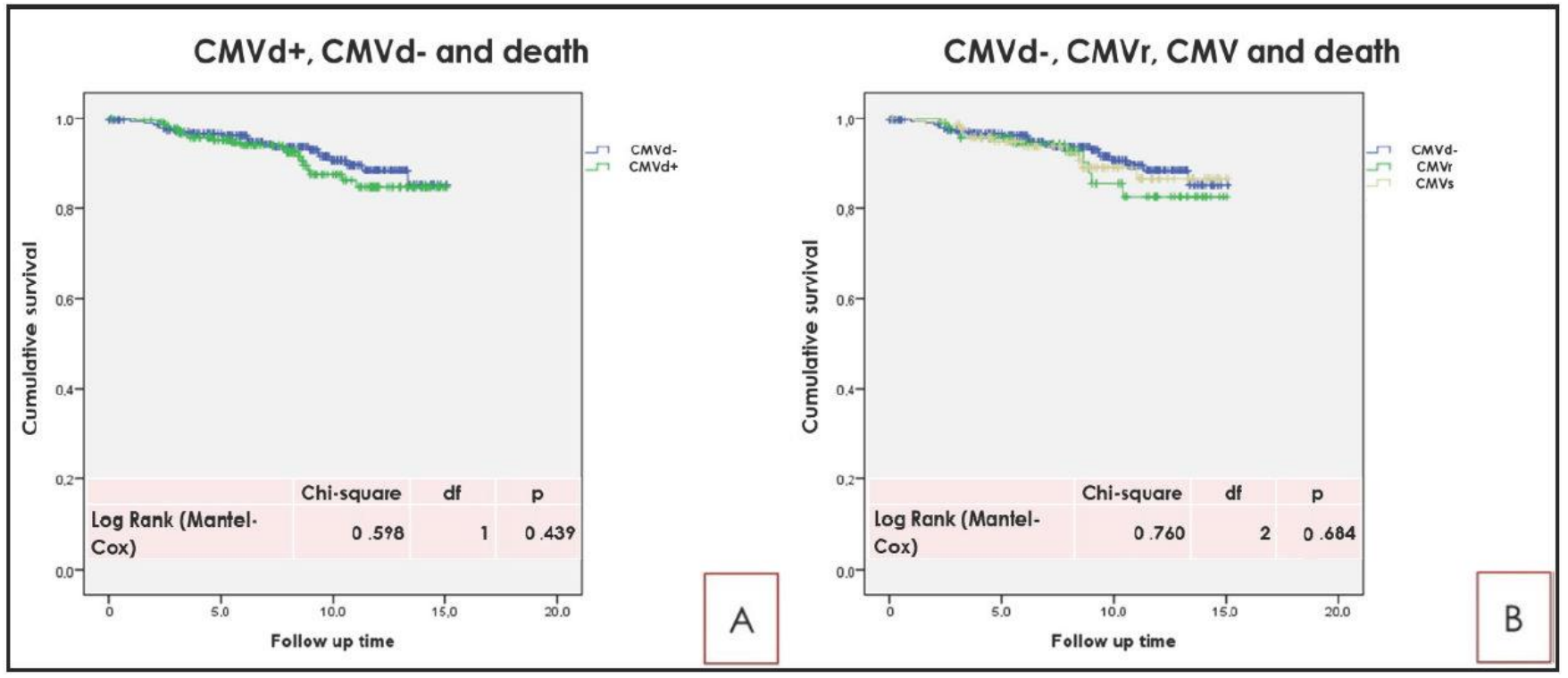

Figure 2. (A): $\mathrm{CMVd}$ and death. (B): $\mathrm{CMVd}-, \mathrm{CMVr}$, and $\mathrm{CMVs}$ and death. As reported in the text, no significant differences for CMVd, CMVr, and CMVs were found according to patient survival. Kaplan Meier survival analysis. The log rank test (Mantel-Cox) was used. 


\section{Discussion}

The first aim of our study was to evaluate CMVd prevalence during the first year of RTx in a cohort of 505 RTx-p.

The prevalence of $\mathrm{CMVd}$ was $45 \%$. Among CMVd+, $43 \%$ experienced a $\mathrm{CMVr}$, whereas in $57 \%$, CMVs was found. These results are in accordance with some data reported in the literature $[10,11]$. In the work presented by Witzcke et al., in which 296 RTx-p were studied, the prevalence of CMVd and CMVr was of $51 \%$ and $75 \%$, respectively [12].

Concerning demographic characteristics, it is important to underscore that, in contrast with the principal international studies, in our cohort, CMVd had a significantly higher prevalence in the female gender. In some reports, a higher CMV female serum positivity in the Italian population has been described compared to the rest of the world. As reported in some Italian multicentric studies, CMV serum positivity in Italian women is $>90 \%[13,14]$. In Europe, this high prevalence was highlighted in Italy, Sweden, and, outside Europe, in Brazil [15].

The results of our study confirm the distribution of CMV serology among RTx-p, with a strong prevalence of the R+/D+ pattern $(74 \%)$, followed by $\mathrm{R}-/ \mathrm{D}+(13 \%), \mathrm{R}+/ \mathrm{D}-(10 \%)$, and $\mathrm{R}-/ \mathrm{D}-(3 \%)$. In our cohort, the serological status mostly correlated with CMVd has been $\mathrm{R}-/ \mathrm{D}+$. In agreement to our results, Kute et al., in a study of $750 \mathrm{RTx}-\mathrm{p}$, indicated the $\mathrm{R}-/ \mathrm{D}+$ status as one of the principal risk factors for the development of CMVd [16]. In addition, Selvey et al. confirmed the independent association between CMV R-/D+ and CMVd with an hazard ratio of 5.44 (2.49-11.89) [17].

Our study also aimed to assess the most impacting factors on CMVd development during the first year of RTx. From the comparison between CMVd+ and CMVd-, age was directly correlated to CMVd+. This could result from different components, partly mutually related. Certainly, a global cellular senescence might influence the general responsiveness of the immune system, as reported in the study of Arthurs et al. [18]. In agreement with this, in another study where 1127 RTx-p were considered, a Charlson comorbidity index $>3$ was strictly correlated to the development (over 3 months after the RTx) of CMVs [18]. Another important factor might be represented by the fact that the allocation to older patients of potentially marginal organs, theoretically more immunogenic, requires stronger immunosuppressive protocols.

In our cohort, CMVd was found more frequently in patients who underwent dialysis independently of dialysis modality and vintage. Several studies have reported a relationship between uremic toxins and oxidative stress. This, in addition, negatively influences the immune responsiveness, and might affect the patient's nutritional and health status [19,20]. As mentioned above, our data showed no correlation between dialysis vintage and CMVd development. The impact of dialysis vintage on CMVd is still debated. In 2002, Abbot et al. evaluated in 33749 RTx-p the factors mostly related to hospitalization for CMV. According to their results, history of dialysis and dialysis vintage were independently associated with the outcome considered [21]. More recently, however, Corivaud et al. found no difference in CMVs frequency in $2010 \mathrm{RTx}$-p regarding dialysis technique and vintage [22].

Another important data from our analyses is the total absence of CMVd in RTx-p transplanted from living donors. A low $\mathrm{CMVd}+$ prevalence was described in the literature in some papers. In particular, in a cohort of 592 RTx-p (214 RTx-p \% and from living donors), Giakoustidis et al. reported a prevalence of $\mathrm{CMVd}+$ of $7 \%$ and $12 \%$ respectively in living and deceased donor RTx [23]. The procedure frequently carried out before the start of replacement therapy, the better donor and transplant status, and the short ischemia time of the transplanted organ might be important factors in explaining this result [24]. Another possible explanation might be derived from the fact that related recipient and donor might have been infected with the same type of CMV, having antibodies against the same CMV serotype. However, in our cohort, most of RTx living donors were non related to recipients.

Interestingly, and in accordance with some reports, $\mathrm{CMVd}+$ patients had higher prevalence of pre-RTx diabetes. In this regard, basic research studies indicate a potential 
promoting role, through a reduction in cell-mediated immunity, for diabetic status in CMVd development [25].

Concerning immunosuppressive therapy, the cumulative dose of steroids was found to be the only related factor in the onset both of CMVr and CMVs. This might be due to the effects of steroids on immunity (influence on activation, proliferation and apoptosis of T-cells; modulating effects on the NF-kb system) and in CMV reactivation mechanism (mediated by the same NF-kb pathway) [26-29].

Finally, in our study, Anti-thymocyte globulin (ATG) induction therapy was not associated with a higher frequency of CMVd. This topic is debated, considering CMV prophylaxis protocols frequently used in ATG treated RTx-p [30,31]. However, the strong lymphocytic depletion derived by the use of these antibodies might theoretically increase the risk of CMV replication [32]. It is important to point out that the prescription of ATG based induction protocols has increased strongly in the last 4-5 years in our Department and this might be a bias on this analysis. Concerning therapy with inhibiting mTORs, a statistically significant difference between CMVd+ and CMVd- was found at T1. In 2011, Brennan et al. reported, in a study of 2004 patients, a significantly reduced incidence of $\mathrm{CMVd}+$ in those who had used high dosages of $\mathrm{m}$-Tor inhibitors [32].

According to our data, one of the strongest and inversely related factors to the development of CMVd is serum albumin. In patients with CKD and RTx, albumin is considered an indirect indicator of inflammatory, as well as nutritional status [33-35]. Basic research data showed that albumin has a scavenger function of various endotoxins and oxidizing factors such as nitric oxide and its relationship with CMVd might be explained by the link between global inflammation and the activation of the NF-kb pathway, which can promote CMV replication [36-38]. Moreover, Liu et al. recently reported that CMV is able to downregulate the neonatal Fc receptor, involved in albumin level maintenance via US11. This might influence the relationship between CMV replication and serum albumin [39].

In addition, some evidence has reported the hypothesis that the pharmacological activity of mycophenolate mofetil (MMF), partially bounded to albumin, is a function of unbound drug concentration. This might imply a higher unbound MMF concentration in those patients with lower albumin levels, and consequently a higher degree of immunosuppression exposition $[40,41]$.

The correlation between serum albumin levels and CMVd in RTx has been recently investigated. Srivastava et al., in their work published in 2020, enrolled 1717 RTx-p with the aim of studying the correlation of pre-transplant albumin levels and CMVd during RTx. In this study, severe pre-RTx hypoalbuminemia was associated with an increase incidence of post RTx CMVd and the dose-response relationship showed a slight decrease in CMVd risk with higher albumin levels [42]. In addition to that, some evidence is reported in liver transplantation. In 2010, Kim et al. studied 353 liver transplant patients for an average follow-up of 13 years. Data obtained in their study showed that patients who were found to have CMV replication had significantly lower values of albumin and $\mathrm{Hb}$. Of note, also in that work, serum albumin was independently and inversely associated to CMVd [43].

Considering the several discordant results present in the literature concerning CMVd and early and long-term RTx and RTx-p outcomes, we decided to also explore this topic. During the FU time, $11 \%$ of RTx-p restarted dialysis, and $8 \%$ died. The incidence of the outcomes considered was significantly lower than the general European and American data. In fact, in Europe, the average survival rate of 5-year transplantation is $80 \%$, with mortality rates always at 5 years of $9 \%$, and in the USA, the 10-year graft survival rate is $35 \%$ and the RTx-p mortality rate, $36 \%$ [44,45].

From the analyses presented in our work, CMVd, CMVr, and CMVs showed no significant association with the major clinical outcomes evaluated. These results are partially in contrast with some data reported in the literature. In particular, Sagedal et al., in 2004, analyzing a cohort of 471 RTx-p for 7 years, found a correlation between CMVd and mortality for all causes, and an impact only of CMVs on graft failure. Differently to the clinical practice of our Department, in that study, none of the patients considered 
received CMV prophylaxis and, after the first 3 months of RTx, patients were reevaluated for CMV-DNA once a year [46]. More recently, another study performed by Aziz et al., in 2020, in 757 kidney-pancreas transplant patients, found a negative correlation between CMV infection and all cause kidney graft survival. It should be noted, however, that in this study, CMV viremia was not checked during CMV prophylaxis unless there was clinical suspicion of CMV disease; moreover, CMVd was not well defined and diagnosis of viral activation was made using a "diagnosis code". Therefore, there was no data on the type and the severity of CMVd in that cohort. On the other hand, two important studies, both by Erdbrugger et al., showed results in a cohort of 594 kidney-pancreas transplant patients that were closely similar with many of our findings. Of note, CMV prophylaxis protocols adopted in those cohorts were almost identical to the one used in our Center. In these studies, the multivariate analyses indicated a strong influence both of higher donor age and of worse early post-RTx graft function in determining graft loss. CMV viremia or disease were not significantly related with graft outcome, detection of chronic rejection, and patient survival $[47,48]$. In addition, in our study, other minor and combined clinical outcomes were also considered in statistical assessments (eGFRr $>50 \%$ to $\mathrm{FU}, \mathrm{sCr}>50 \%$ to $\mathrm{FU}$, outcome combined $\mathrm{sCr}>50 \%-\mathrm{D}+$ and eGFRr $>50 \%-\mathrm{D}+$, and overall reduction of eGFR weighted for the time of FU), but no correlations with CMV were found.

Our study presents, as its principal limitations, the monocentric and the retrospective observational design and this has not allowed the evaluation of any causal relationships derived from the demonstrated associations and a more detailed investigation concerning the relationship between CMVd and inflammatory status (IL-6, MCP-1, etc.). Moreover, the fact that different dosage methods were adopted for CMV-DNA viral load from 2004 to 2017 in our Laboratory did not allow a focused statistical analysis. However, the high number of patients considered, and the relatively long FU time are, in our opinion, important features. In any case, this work might promote future randomized and prospective studies on the topics proposed.

In conclusion, our study demonstrated a relatively high prevalence of CMVd during the first year of RTx. Serum albumin at T1 was the most important independent factor which could influence CMVd insurgence. Nevertheless, CMVd did not show any relationship with principal RTx and RTx-p outcomes.

\section{Materials and Methods}

\subsection{Study Design and Characteristics}

In our study, data concerning 505 RTx-p were collected. The cohort studied was selected, among the 616 RTx-p transplanted in our Department between January 2004 and December 2016, according to the availability both of donor (D) and recipient (R) CMV serology at the moment of RTx and the achieving of one year RTx follow-up (6 and 2 patients restarted dialysis and died before the 12th month of RTx). The studied cohort, followed up for a minimum time of 12 months and a global median time of 8.3 years, was similar in the main clinical and biochemical characteristics to the one excluded from the study.

At RTx, for each of the 505 RTx-p examined, the following data were considered:General characteristics: age, gender, dialysis vintage and technique, native renal disease, history of diabetes mellitus before RTx, steroid use before RTx, hepatitis C virus serology, cold ischemia time, type of RTx (living/deceased donor);-At 1st (T1) and 12th (T12) month after RTx: body weight, systolic and diastolic blood pressure; routine laboratory assessment including serum creatinine ( $\mathrm{sCr}$ ), estimation of glomerular filtration rate (eGFR), hemoglobin $(\mathrm{Hb})$, serum albumin, blood glucose, glycated hemoglobin $(\mathrm{HbA} 1 \mathrm{C})$, uric acid, C-reactive protein (CRP), 24-h protein urinary excretion (Prot-U);- - In addition, data about: immunosuppressive therapy regimens were evaluated at $\mathrm{T} 1$ and T12; steroid therapy was considered as total drug exposure, in $\mathrm{mg}$, at $\mathrm{T} 1$ and $\mathrm{T} 12$.

Urinary and blood evaluations were performed after $12 \mathrm{~h}$ of fasting at the Transplant Clinic of Our Department and analyzed by the Central Laboratory of our Hospital. 
All other biochemical parameters were measured according to the routine methodology used in our central laboratory. The $\mathrm{sCr}$ assessed was done by Jaffe's reaction, whereas eGFR was estimated using the Modification of Diet in Renal Disease (MDRD) formula [49]. Urinary protein excretion was assessed by measuring the 24-h urinary collection protein through the immunoturbidimetric method.

Given the observational and retrospective nature of the study, it was not necessary to obtain informed consent from patients. In any case, all the data were collected digitally, analyzed, and reported in the results in a totally anonymous manner.

\subsection{Cytomegalovirus Analysis}

According to CMV IgG serology, assessed at the moment of RTx both in R and D, four groups for CMV serology were defined: recipient CMV IgG-/donor CMV IgG(CMV R-/D-); recipient CMV IgG-/donor CMV IgG+ (CMV R-/D+); recipient CMV $\mathrm{IgG}+$ /donor CMV IgG- (CMV R+/D-); recipient CMV IgG+/donor CMV IgG+ (CMV $\mathrm{R}+/ \mathrm{D}+)$.

In agreement with the clinical practice of our Department, prophylactic therapy for $\mathrm{CMV}$, using valganciclovir at dosage corrected for renal function, was administered during the first three months after RTx to those patients considered at high risk of CMVd (CMV $\mathrm{R}-/ \mathrm{D}+$, patients treated with ATG induction therapy, previous history of RTx).

During the first year of RTx in all the cohort, CMV viremia (CMV-DNA) was tested in all the RTx-p with the following frequency: within the first month of RTx: one to three times a week; from two to four months of RTx: every 10 days; from 4 to 6 months of RTx: every 15-20 days; from six months to 12 months after RTx: monthly.

According to the different dosage methods adopted for CMV-DNA from 2004 to 2017, the following cut-off values of CMV-DNA were used to define the presence of CMVd: from 2004 to 2006: CMV-DNA > 40 copies; from 2007 to the end of 2016: CMV-DNA > 500 copies; from 2017: CMV-DNA > 135 copies.

Patients with positive CMV-DNA, in the absence of clinical and biochemical signs of disease and in which no modifications of immunosuppressive and antiviral therapy were necessary, were defined as replicant CMVd (CMVr). Patients with positive CMV-DNA, associated to CMV clinical and/or biochemical signs of disease (for instance CMV related leucopenia and/or increase of liver necrosis indices) and in which a reduction of immunosuppressive therapy (for instance mycophenolate suspension) and/or a treatment with specific antiviral drugs were prescribed, have been defined as symptomatic CMVd (CMVs).

\subsection{Outcomes and Follow up}

Patients were followed up for a median time of 8.3 (5.4-11.4) years.

At the end of follow up (FU), the following outcomes were evaluated: increase of $\mathrm{sCr}$ $>50 \%$ compared to $\mathrm{T} 1(\mathrm{sCr}>50 \%$ ); reduction of eGFR $>50 \%$ compared to $\mathrm{T} 1$ (eGFR $>50 \%$ ); reduction of eGFR/year: $(\mathrm{mL} / \mathrm{min}) /$ year; Graft loss, defined by the need of restart of dialysis; RTx-p death. The following combined outcomes were also considered: graft loss or increase in $\mathrm{sCr}>50 \%$ compared to $\mathrm{T} 1$; graft loss or eGFR $>50 \%$ reduction compared to T1.

\subsection{Statistical Analyses}

Continuous variables were expressed as median (25-75\% ile). Differences between the groups were determined using the Student $t$-Test, the Wilcoxon-Mann-Whitney test, Kruskal Walls, ANOVA, the Chi square test, and the Fisher test when indicated. Logistic regression models were performed for multivariate analyses. The Kaplan-Meier test with $\log$ rank significance tests was used for survival analyses. Statistical significance was set for $p<0.05$ values. Statistical analyses were performed using Statistica version 10 and SPSS 20. 
Author Contributions: P.M. (Piergiorgio Messa) was responsible for study concept and design; C.M.A., P.M. (Paolo Molinari), M.G., M.C., D.C., A.R., M.L., M.I., S.D. and E.F. wrote the main manuscript; C.M.A. and P.M. (Paolo Molinari) performed the statistical analyses. All authors have read and agreed to the published version of the manuscript.

Funding: This research received no external funding.

Institutional Review Board Statement: All procedures performed in studies involving human participants were in accordance with the ethical standards of the institutional and/or national research committee and with the 1964 Helsinki declaration and of the Declaration of Istanbul and its later amendments or comparable ethical standards.

Informed Consent Statement: Given the observational and retrospective nature of the study, it was not necessary to obtain informed consent from patients. In any case, all the data were collected digitally, analyzed, and reported in the results in a totally and anonymous manner.

Data Availability Statement: The data presented in this study are available on request from the corresponding author. The data are not publicly available due to privacy issues.

Acknowledgments: The authors thank ssa Marina Balderacchi for the kind collaboration in the health research and in the realization of this study.

Conflicts of Interest: The authors declare no conflict of interest.

\section{References}

1. Coresh, J.; Selvin, E.; Stevens, L.A.; Manzi, J.; Kusek, J.W.; Eggers, P.; Van Lente, F.; Levey, A.S. Prevalence of Chronic Kidney Disease in the United States. JAMA 2007, 298, 2038. [CrossRef]

2. $\quad$ Brück, K.; Stel, V.S.; Gambaro, G.; Hallan, S.; Völzke, H.; Ärnlöv, J.; Jager, K.J. Clinical epidemiology ckd Prevalence Varies across the European General Population. J. Am. Soc. Nephrol. 2016, 27, 2135-2147. [CrossRef]

3. Silkensen, J.R. Long-Term Complications in Renal Transplantation. J. Am. Soc. Nephrol. 2000, 11, 582-588.

4. Ramanan, P.; Razonable, R.R. Cytomegalovirus infections in solid organ transplantation: A review. Infect. Chemother. 2013, 45, 260-271. [CrossRef]

5. Karuthu, S.; Blumberg, E.A. Common Infections in Kidney Transplant Recipients. Clin. J. Am. Soc. Nephrol. 2012, 7, 2058-2070. [CrossRef] [PubMed]

6. $\quad$ Ljungman, P.; Boeckh, M.; Hirsch, H.H.; Josephson, F.; Lundgren, J.; Nichols, G.; Pikis, A.; Razonable, R.R.; Miller, V.; Griffiths, P.D. Definitions of Cytomegalovirus Infection and Disease in Transplant Patients for Use in Clinical Trials: Table 1. Clin. Infect. Dis. 2017, 64, 87-91. [PubMed]

7. Razonable, R.R.; Humar, A. Cytomegalovirus in solid organ transplant recipients-Guidelines of the American Society of Transplantation Infectious Diseases Community of Practice. Clin. Transplant. 2019, 33, e13512. [CrossRef] [PubMed]

8. Kotton, C.N.; Kumar, D.; Caliendo, A.M.; Huprikar, S.; Chou, S.; Danziger-Isakov, L.; Humar, A. The Third International Consensus Guidelines on the Management of Cytomegalovirus in Solid-organ Transplantation. Transplantation 2018, 102, 900-931. [CrossRef]

9. Linares, L.; Sanclemente, G.; Cervera, C.; Hoyo, I.; Cofán, F.; Ricart, M.; Pérez-Villa, F.; Navasa, M.; Marcos, M.; Antón, A.; et al. Influence of Cytomegalovirus Disease in Outcome of Solid Organ Transplant Patients. Transplant. Proc. 2011, 43, 2145-2148. [CrossRef]

10. Meesing, A.; Razonable, R.R. New Developments in the Management of Cytomegalovirus Infection after Transplantation. Drugs 2018, 78, 1085-1103. [CrossRef]

11. Eid, A.J.; Razonable, R.R. New Developments in the Management of Cytomegalovirus Infection after Solid Organ Transplantation. Drugs 2010, 70, 965-981. [CrossRef]

12. Witzke, O.; Hauser, I.A.; Bartels, M.; Wolf, G.; Wolters, H.; Nitschke, M. Valganciclovir Prophylaxis Versus Preemptive Therapy in Cytomegalovirus-Positive Renal Allograft Recipients: 1-Year Results of a Randomized Clinical Trial. Transplantation 2012, 93, 61-68. [CrossRef]

13. Volpi, A.; Pica, F.; Cauletti, M.; Panà, A.; Rocchi, G. Cytomegalovirus infection in day care centers in Rome, Italy: Viral excretion in children and occupational risk among workers. J. Med. Virol. 1988, 26, 119-125. [CrossRef] [PubMed]

14. Barbi, M.; Binda, S.; Caroppo, S.; Calvario, A.; Germinario, C.; Bozzi, A.; Tanzi, M.L.; Veronesi, L.; Mura, I.; Piana, A.; et al. Multicity Italian study of congenital cytomegalovirus infection. Pediatr. Infect. Dis. J. 2006, 25, 156-159. [CrossRef] [PubMed]

15. Cannon, M.J.; Schmid, D.S.; Hyde, T.B. Review of cytomegalovirus seroprevalence and demographic characteristics associated with infection. Rev. Med. Virol. 2010, 20, 202-213. [CrossRef] [PubMed]

16. Kute, V.B.; Vanikar, A.V.; Shah, P.R.; Gumber, M.; Patel, H.; Godara, S.; Munjappa, B.; Sainaresh, V.; Engineer, D.; Jain, S.; et al. Post-Renal Transplant Cytomegalovirus Infection: Study of Risk Factors. Transplant. Proc. 2012, 44, 706-709. [CrossRef] [PubMed] 
17. Selvey, L.A.; Lim, W.H.; Boan, P.; Swaminathan, R.; Slimings, C.; Harrison, A.E.; Chakera, A. Cytomegalovirus viraemia and mortality in renal transplant recipients in the era of antiviral prophylaxis. Lessons from the western Australian experience. BMC Infect. Dis. 2017, 17, 501. [CrossRef]

18. Arthurs, S.K.; Eid, A.J.; Pedersen, R.A.; Kremers, W.K.; Cosio, F.G.; Patel, R.; Razonable, R.R. Delayed-Onset Primary Cytomegalovirus Disease and the Risk of Allograft Failure and Mortality after Kidney Transplantation. Clin. Infect. Dis. 2008, 46, 840-846. [CrossRef]

19. Cohen, G.; Hörl, W.H. Immune Dysfunction in Uremia-An Update. Toxins 2012, 4, 962-990. [CrossRef]

20. Kalantar-Zadeh, K.; Kilpatrick, R.D.; Kuwae, N.; McAllister, C.J.; Alcorn, H.; Kopple, J.D.; Greenland, S. Revisiting mortality predictability of serum albumin in the dialysis population: Time dependency, longitudinal changes and population-attributable fraction. Nephrol. Dial. Transplant. 2005, 20, 1880-1888. [CrossRef]

21. Abbott, K. Hospitalizations for Cytomegalovirus Disease after Renal Transplantation in the United States. Ann. Epidemiol. 2002, 12, 402-409. [CrossRef]

22. Courivaud, C.; Ladriere, M.; Toupance, O.; Caillard, S.; De Ligny, B.H.; Ryckelynck, J.-P.; Moulin, B.; Rieu, P.; Frimat, L.; Chalopin, J.-M.; et al. Impact of pre-transplant dialysis modality on post-transplant diabetes mellitus after kidney transplantation. Clin. Transplant. 2011, 25, 794-799. [CrossRef]

23. Giakoustidis, D.; Antoniadis, A.; Fouzas, I.; Sklavos, A.; Ouzounidis, N.; Gakis, D.; Koubanagiti, K.; Myserlis, G.; Tsitlakidis, A.; Gerogiannis, I.; et al. Prevalence and clinical impact of cytomegalovirus infection and disease in renal transplantation: Ten years of experience in a single center. Transplant. Proc. 2012, 44, 2715-2717. [CrossRef]

24. Davis, C.L.; Delmonico, F.L. Living-Donor Kidney Transplantation: A Review of the Current Practices for the Live Donor. J. Am. Soc. Nephrol. 2005, 16, 2098-2110. [CrossRef]

25. Geerlings, S.E.; Hoepelman, A.I.M. Immune Dysfunction in Patients with Diabetes Mellitus. FEMS Immunol. Med Microbiol. 1999, 26, 259-265. [CrossRef] [PubMed]

26. Tandon, R.; Mocarski, E.S. Viral and host control of cytomegalovirus maturation. Trends Microbiol. 2012, 20, 392-401. [CrossRef] [PubMed]

27. De Keyzer, K.; Van Laecke, S.; Peeters, P.; Vanholder, R. Human Cytomegalovirus and Kidney Transplantation: A Clinician's Update. Am. J. Kidney Dis. 2011, 58, 118-126. [CrossRef] [PubMed]

28. Barnes, P.J. How corticosteroids control inflammation: Quintiles Prize Lecture 2005. Br. J. Pharmacol. 2006, 148, 245-254. [CrossRef]

29. Halloran, P.F. Immunosuppressive Drugs for Kidney Transplantation. N. Engl. J. Med. 2004, 351, 2715-2729. [CrossRef]

30. Couzi, L.; Helou, S.; Bachelet, T.; Martin, S.; Moreau, K.; Morel, D.; Lafon, M.E.; Garrigue, I.; Merville, P. Preemptive therapy versus valgancyclovir prophylaxis in cytomegalovirus-positive kidney transplant recipients receiving antithymocyte globulin induction. Transplant. Proc. 2012, 44, 2809-2813. [CrossRef]

31. Issa, N.C.; Fishman, J.A. Infectious Complications of Antilymphocyte Therapies in Solid Organ Transplantation. Clin. Infect. Dis. 2009, 48, 772-786. [CrossRef]

32. Brennan, D.C.; Legendre, C.; Patel, D.; Mange, K.; Wiland, A.; McCague, K.; Shihab, F.S. Cytomegalovirus Incidence Between Everolimus Versus Mycophenolate in De Novo Renal Transplants: Pooled Analysis of Three Clinical Trials. Am. J. Transplant. 2011, 11, 2453-2462. [CrossRef] [PubMed]

33. Obi, Y.; Qader, H.; Kovesdy, C.P.; Kalantar-Zadeh, K. Latest consensus and update on protein-energy wasting in chronic kidney disease. Curr. Opin. Clin. Nutr. Metab. Care 2015, 18, 254-262. [CrossRef]

34. Soriano, S.; González, L.; Martín-Malo, A.; Rodríguez, M.; Aljama, P. C-reactive protein and low albumin are predictors of morbidity and cardiovascular events in chronic kidney disease (CKD) 3-5 patients. Clin. Nephrol. 2007, 67, 352-357. [CrossRef] [PubMed]

35. van Ree, R.M.; Gross, S.; Zelle, D.M.; van der Heide, J.J.H.; Schouten, J.; van Son, W.J.; Gans, R.O.B.; Bakker, S.J.L. Influence of C-Reactive Protein and Urinary Protein Excretion on Prediction of Graft Failure and Mortality by Serum Albumin in Renal Transplant Recipients. Transplantation 2010, 89, 1247-1254. [CrossRef] [PubMed]

36. Levitt, D.G.; Levitt, M.D. Human serum albumin homeostasis: A new look at the roles of synthesis, catabolism, renal and gastrointestinal excretion, and the clinical value of serum albumin measurements. Int. J. Gen. Med. 2016, 9, 229. [CrossRef] [PubMed]

37. Evans, T.W. Review article: Albumin as a drug-biological effects of albumin unrelated to oncotic pressure. Aliment Pharmacol. Ther. 2002, 16, 6-11. [CrossRef] [PubMed]

38. Mocarski, E.S. Immune escape and exploitation strategies of cytomegaloviruses: Impact on and imitation of the major histocompatibility system. Cell Microbiol. 2004, 6, 707-717. [CrossRef]

39. Liu, X.; Palaniyandi, S.; Zhu, I.; Tang, J.; Li, W.; Wu, X.; Ochsner, S.P.; Pauza, C.D.; Cohen, J.I.; Zhu, X. Human cytomegalovirus evades antibody-mediated immunity through endoplasmic reticulum-associated degradation of the FcRn receptor. Nat. Commun. 2019, 10, 3020. [CrossRef]

40. Nowak, I.; Shaw, L.M. Mycophenolic acid binding to human serum albumin: Characterization and relation to pharmacodynamics. Clin. Chem. 1995, 41, 1011-1017. [CrossRef]

41. Ma, X.; Yan, J.; Wang, Q.; Wu, D.; Li, H. Spectroscopy study and co-administration effect on the interaction of mycophenolic acid and human serum albumin. Int. J. Biol. Macromol. 2015, 77, 280-286. [CrossRef] [PubMed] 
42. Srivastava, A.; Bodnar, J.; Osman, F.; Jorgenson, M.R.; Astor, B.C.; Mandelbrot, D.A.; Parajuli, S. Serum Albumin Level Before Kidney Transplant Predicts Post-transplant BK and Possibly Cytomegalovirus Infection. Kidney Int. Rep. 2020, 5, $2228-2237$. [CrossRef] [PubMed]

43. Kim, J.M.; Kim, S.J.; Joh, J.-W.; Shin, M.; Moon, J.; Jung, G.; Choi, G.-S.; Kwon, C.; Lee, S.-K. The Risk Factors for Cytomegalovirus Syndrome and Tissue-invasive Cytomegalovirus Disease in Liver Transplant Recipients Who Have Cytomegalovirus Antigenemia. Transplant. Proc. 2010, 42, 890-894. [CrossRef] [PubMed]

44. USRDS 2018-Chapter 6: Transplantation. Available online: https://www.usrds.org/annual-data-report/ (accessed on 14 March 2021).

45. Zoccali, C.; Massy, Z.; Caskey, F.J.; Couchoud, C.; Evans, M.; Finne, P.; Groothoff, J.W.; Harambat, J.; Heaf, J.G.; Jarraya, F.; et al. ERA-EDTA Registry Annual Report 2016; ERA-EDTA Registry: Amsterdam, The Netherlands, 2018.

46. Sagedal, S.; Hartmann, A.; Nordal, K.P.; Osnes, K.; Leivestad, T.; Foss, A.; Degré, M.; Fauchald, P.; Rollag, H. Impact of early cytomegalovirus infection and disease on long-term recipient and kidney graft survival. Kidney Int. 2004, 66, 329-337. [CrossRef]

47. Erdbruegger, U.; Scheffner, I.; Mengel, M.; Schwarz, A.; Verhagen, W.; Haller, H.; Gwinner, W. Impact of CMV infection on acute rejection and long-term renal allograft function: A systematic analysis in patients with protocol biopsies and indicated biopsies. Nephrol. Dial. Transplant. 2012, 27, 435-443. [CrossRef] [PubMed]

48. Erdbrügger, U.; Scheffner, I.; Mengel, M.; Schwarz, A.; Haller, H.; Gwinner, W. Long-term impact of CMV infection on allografts and on patient survival in renal transplant patients with protocol biopsies. Am. J. Physiol. Ren. Physiol. 2015, 309, F925-F932. [CrossRef] [PubMed]

49. The Modification of Diet in Renal Disease Study: Design, methods, and results from the feasibility study. Am. J. Kidney Dis. 1992, 20, 18-33. [CrossRef] 